\title{
Nonparametric Rank Tests for Independence in Opinion Surveys
}

\author{
Philip L.H. $\mathrm{Yu}^{+}$, K.F. Lam ${ }^{+}$, and Mayer Alvo* \\ + The University of Hong Kong \\ * University of Ottawa, Canada
}

\begin{abstract}
Nonparametric rank tests for independence between two characteristics are commonly used in many social opinion surveys. When both characteristics are ordinal in nature, tests based on rank correlations such as those due to Spearman and Kendall are often used. The case where some ties exist has already been considered whereas Alvo and Cabilio (1995) have studied the case when there are missing values but no ties in the record. However, it frequently happens that the survey data may contain simultaneously many tied observations and/or many missing values. A naive approach is to simply discard the missing observations and then to make use of the rank correlations adjusted for ties. This approach would be less powerful as it does not fully utilize the information associated with the incomplete data set. In this article, we generalize Alvo and Cabilio's notion of distance between two rankings to incorporate tied and missing observations, and define new test statistics based on the Spearman and Kendall rank correlation coefficients. We determine the asymptotic distribution of the Spearman test statistic and compare its efficiency with the corresponding statistic based on the naive approach. The proposed test is then applied to a real data set collected from an opinion survey conducted in Hong Kong.
\end{abstract}

Keywords: Opinion Surveys; Asymptotic Relative Efficiency; Incomplete Rankings; Ties; Rank Correlation; Spearman and Kendall Distances.

\section{Introduction}

Discrete ordinal variables are very popularly seen in many social opinion surveys. To test for the independence between any two such variables, a nonparametric test based on Spearman rank correlation is commonly used. However, it frequently happens that the survey data may contain some missing values. For example in a public opinion survey carried out in early 1999 in Hong Kong by the Social Science Research Centre of the University of Hong Kong, it was of interest to determine whether the age of the respondents is related to the level of satisfaction of the Policy Address of the Chief Executive of the Hong Kong Special Administrative Region. The response is an ordinal variable with the seven options being: 1 - very satisfied, 2 - satisfied, 3 - neutral, 4 - unsatisfied, 5 - very unsatisfied, 6 - not sure, and 7 - refuse to answer. Options 6 and 7 will be classified as missing (non-response). The age at last birthday of the respondents was recorded when available. The following table shows the frequencies of female respondents classified by their responses and age groups.

It can be seen from Table 1 that the problem of missing values is quite severe, about $29.3 \%$ of respondents did not respond on either one or both questions. A naive approach 
Table 1: Data from the public opinion survey

\begin{tabular}{|c|c|c|c|c|c|c|c|}
\hline & \multicolumn{6}{|c|}{ Age Group } & \\
\hline Response & $18-24$ & $25-34$ & $35-44$ & $45-54$ & $>54$ & Missing & Sub-total \\
\hline 1 & 0 & 0 & 0 & 0 & 0 & 0 & 0 \\
\hline 2 & 10 & 12 & 17 & 16 & 10 & 5 & 70 \\
\hline 3 & 16 & 29 & 37 & 21 & 3 & 7 & 113 \\
\hline 4 & 8 & 9 & 10 & 7 & 1 & 3 & 38 \\
\hline 5 & 1 & 1 & 3 & 1 & 0 & 0 & 6 \\
\hline Missing & 10 & 14 & 19 & 10 & 14 & 6 & 73 \\
\hline Sub-Total & 45 & 65 & 86 & 55 & 28 & 21 & 300 \\
\hline
\end{tabular}

is to simply discard the missing observations and make use of the classical Spearman rank test for testing the independence between the two variables (see Lehmann, 1975, p. 301). Clearly, this approach would be less powerful as it does not utilize the information associated with the incomplete data set. Since the responses and the ages of the respondents are classified into a few categories, the problem of ties is also very severe. Another approach consists of analyzing the data as a contingency table. However, in that case, the natural ordering which exists among the age groups and similarly among the responses would not be used. The objective of this paper is to develop rank tests for testing independence between two ordinal variables which can incorporate the presence of both missing values and ties. Only tests based on Spearman and Kendall rank correlations will be considered here.

Rank-based correlations due to Spearman and Kendall play an important role as measures of association between two factors and as tests of independence between two random variables. When the data contain missing observations but no ties, Alvo and Cabilio (1995) proposed a new class of rank correlations based on the concept of distance between rankings and derived the corresponding asymptotic distributions of the test statistics. However, their method could not be directly applied to the above-mentioned two-way ordinal classification problem as the data contain many ties.

Suppose in a group of $t$ respondents, each respondent is asked to assign scores on two characteristics, say A and B. Ranking the respondents according to their scores (called objects hereafter) on characteristics $\mathrm{A}$ and $\mathrm{B}$ results in two complete rankings of $t$ objects $\boldsymbol{\mu}=(\mu(1), \mu(2), \cdots, \mu(t))$ and $\boldsymbol{\nu}=(\nu(1), \nu(2), \cdots, \nu(t))$, which can be viewed as permutations of the integers $(1,2, \cdots, t)$, if there are no ties and missing values. The Spearman distance between $\boldsymbol{\mu}$ and $\boldsymbol{\nu}$ given by

$$
d_{S}(\boldsymbol{\mu}, \boldsymbol{\nu})=\frac{1}{2} \sum_{i=1}^{t}(\mu(i)-\nu(i))^{2}
$$

can also be expressed in terms of a similarity measure $A_{S}$

$$
d_{S}(\boldsymbol{\mu}, \boldsymbol{\nu})=c_{S}-A_{S}(\boldsymbol{\mu}, \boldsymbol{\nu})
$$


where

$$
c_{S}=\frac{t\left(t^{2}-1\right)}{12}, \quad A_{S}(\boldsymbol{\mu}, \boldsymbol{\nu})=\sum_{i=1}^{t}\left(\mu(i)-\frac{t+1}{2}\right)\left(\nu(i)-\frac{t+1}{2}\right) .
$$

The Spearman rank correlation $\rho_{S}$ (Spearman, 1904) can be expressed in terms of $c_{S}$ and $A_{S}$ as $\rho_{S}(\boldsymbol{\mu}, \boldsymbol{\nu})=\frac{A_{S}(\boldsymbol{\mu}, \boldsymbol{\nu})}{c_{S}}$. In an analogous manner, one may define the Kendall rank correlation with distance

$$
d_{K}(\boldsymbol{\mu}, \boldsymbol{\nu})=\sum_{i<j}\{1-\operatorname{sgn}(\mu(i)-\mu(j)) \operatorname{sgn}(\nu(i)-\nu(j))\}
$$

where $\operatorname{sgn}(x)$ is either 1 or -1 depending on whether $x>0$ or $x<0$. In fact, the Kendall rank correlation (Kendall, 1938) becomes $\rho_{K}(\boldsymbol{\mu}, \boldsymbol{\nu})=\frac{A_{K}(\boldsymbol{\mu}, \boldsymbol{\nu})}{c_{K}}$ where

$$
c_{K}=\frac{t(t-1)}{2}, \quad A_{K}(\boldsymbol{\mu}, \boldsymbol{\nu})=\sum_{i<j}\{\operatorname{sgn}(\mu(i)-\mu(j)) \operatorname{sgn}(\nu(i)-\nu(j))\} .
$$

A detailed and complete review of the distance based approach to the analysis of rank data is given in Alvo and Cabilio (1993).

In the next section, we recall the notion of compatibility of Alvo and Cabilio (1995) and use it in Section 3, to introduce new test statistics based on the Spearman and Kendall distances when both ties and missing observations are present. The proposed tests specialize to the known test statistics of Alvo and Cabilio (1995) when there are only missing observations and to the classical Spearman and Kendall tests when only ties are present. The asymptotic distribution of the new Spearman test statistic is derived. It is also found that the two proposed tests are asymptotically equivalent. Some remarks on the asymptotic efficiency of the new Spearman test are made. In Section 4, we apply the new Spearman test to the above opinion survey data. We conclude with some remarks in Section 5.

\section{Extensions to Incomplete Rankings with Ties}

In this section, we propose two new test statistics based on the Spearman and Kendall distances which make use of all the data. First of all, we introduce two separate definitions of compatibility for missing and tied data.

Definition 1 A complete ranking of tobjects is said to be compatible with an incomplete ranking of a subset of $k$ of these objects, $2 \leq k \leq t$ if every pair of the specified $k$ objects is given the same relative ranking in both rankings.

A tied ordering of $t$ objects is partitioned into $e$ sets, $1 \leq e \leq t$, each containing $g_{i}$ objects, $g_{1}+g_{2}+\cdots+g_{e}=t$, so that the $g_{i}$ objects in each set share the rank $\sum_{j=1}^{i-1} g_{j}+g_{i}\left(g_{i}+1\right) / 2,1 \leq i \leq e$. Such a tie pattern is denoted by $\delta=\left(g_{1}, g_{2}, \ldots, g_{e}\right)$.

Definition 2 A complete ranking of tobjects is said to be compatible with a tied ranking of these objects with tie pattern $\delta=\left(g_{1}, g_{2}, \ldots, g_{e}\right)$ if every pair of objects which receive distinct ranks is given the same relative ranking in both rankings. 
The definition of compatibility when there are both ties and missing values is then a blend of the two previous definitions. We shall denote an incomplete ranking of $k$ out of $t$ objects with tie pattern $\delta$ by $\boldsymbol{\mu}^{*}=\left(\mu^{*}(1), \mu^{*}(2), \cdots, \mu^{*}(k)\right)$. At times, this $k$-vector is written as a $t$-vector in which the missing ranks are denoted by the symbol ', . In the presence of ties, the concept of compatibility suggests that $\mu^{*}(i)$ is defined as the midrank of all the items tied with item $i$. We shall denote by $C_{\delta}\left(\boldsymbol{\mu}^{*}\right)$ the class of complete rankings compatible with ranking $\boldsymbol{\mu}^{*}$.

Example. The observations $X_{1}=X_{2}<X_{3}$ and $X_{4}$ is missing yield $t=4, k=3$, $e=2, g_{1}=2, g_{2}=1$ and the incomplete tied ranking written as a 4-vector becomes $\boldsymbol{\mu}^{*}=\left(1.5,1.5,3,_{-}\right)$. The tied ranking $\left(1.5,1.5,3,_{-}\right)$can be viewed as the average of $2 ! 1 !=2$ possible incomplete rankings namely, $\left(1,2,3,,_{-}\right)$and $\left(2,1,3,,_{-}\right)$. The $4 ! / 3 !=4$ complete rankings compatible to $\left(1,2,3,_{-}\right)$are $(1,2,3,4),(1,2,4,3),(1,3,4,2)$ and $(2,3,4,1)$ while the 4 complete rankings compatible to $\left(2,1,3,,_{-}\right)$are $(2,1,3,4),(2,1,4,3),(3,1,4,2)$ and $(3,2,4,1)$. The total $4 ! 2 ! 1 ! / 3 !=8$ complete rankings are then compatible with $\left(1.5,1.5,3,{ }_{-}\right)$.

The notion of distance of Alvo and Cabilio (1995) can be generalized to include both missing and tied rankings as follows.

Definition 3 The Generalized Distance Let $\boldsymbol{\mu}^{*} \quad\left(\boldsymbol{\nu}^{*}\right)$ be an incomplete ranking of $k_{1}$ $\left(k_{2}\right)$ out of $t$ objects with tie pattern $\delta_{1}=\left(g_{11}, g_{12}, \cdots, g_{1 e_{1}}\right)\left(\delta_{2}=\left(g_{21}, g_{22}, \cdots, g_{2 e_{2}}\right)\right)$. The distance between two incomplete rankings $\boldsymbol{\mu}^{*}$ and $\boldsymbol{\nu}^{*}$ is defined to be the average of all distances $d(\boldsymbol{\mu}, \boldsymbol{\nu})$ taken over all pairs of complete rankings $\boldsymbol{\mu}_{i}$ and $\boldsymbol{\nu}_{j}$, compatible with $\boldsymbol{\mu}^{*}$ and $\boldsymbol{\nu}^{*}$, respectively. More formally, let $\kappa_{1}=t !\left(g_{11} ! g_{12} ! \cdots g_{1 e_{1}} !\right) / k_{1} !$ and $\kappa_{2}=$ $t !\left(g_{21} ! g_{22} ! \cdots g_{2 e_{2}} !\right) / k_{2}$ ! be the total number of complete $t$-rankings compatible to $\boldsymbol{\mu}^{*}$ and $\nu^{*}$ respectively, and set $\kappa=\kappa_{1} \kappa_{2}$. Then we have

$$
d^{*}\left(\boldsymbol{\mu}^{*}, \boldsymbol{\nu}^{*}\right)=\frac{1}{\kappa} \sum_{\boldsymbol{\mu}_{i} \in \mathcal{C}_{\delta_{1}}\left(\mu^{*}\right)} \sum_{\boldsymbol{\nu}_{j} \in \mathcal{C}_{\delta_{2}}\left(\nu^{*}\right)} d\left(\boldsymbol{\mu}_{i}, \boldsymbol{\nu}_{j}\right) .
$$

It follows from Alvo and Cabilio (1995) that the generalized Spearman distance $d_{S}^{*}\left(\boldsymbol{\mu}^{*}, \boldsymbol{\nu}^{*}\right)$ can be expressed in terms of a similarity measure $A_{S}$ as

$$
d_{S}^{*}\left(\boldsymbol{\mu}^{*}, \boldsymbol{\nu}^{*}\right)=c_{S}-A_{S}^{*}\left(\boldsymbol{\mu}^{*}, \boldsymbol{\nu}^{*}\right)
$$

with

$$
A_{S}^{*}\left(\boldsymbol{\mu}^{*}, \boldsymbol{\nu}^{*}\right)=\frac{(t+1)^{2}}{\left(k_{1}+1\right)\left(k_{2}+1\right)} \sum_{j=1}^{t} \delta(j)\left[\mu^{*}(j)-\frac{k_{1}+1}{2}\right]\left[\nu^{*}(j)-\frac{k_{2}+1}{2}\right]
$$

where $\delta(j)=1$ if both rankings of item $j$ are not missing, or 0 otherwise. Similarly, it is readily seen that the generalized Kendall similarity measure is given by

$$
A_{K}^{*}\left(\boldsymbol{\mu}^{*}, \boldsymbol{\nu}^{*}\right)=\sum_{i<j} a_{1}(i, j) a_{2}(i, j)
$$

where the $a_{1}(i, j)$ 's are the scores for ranking 1 given by

$$
a_{1}(i, j)= \begin{cases}0 & \text { in case of a tie } \\ 1-\frac{2 \mu^{*}(i)}{k_{1}+1} & \delta(i)=1, \delta(j)=0 \\ \frac{2 \mu^{*}(j)}{k_{1}+1}-1 & \delta(i)=0, \delta(j)=1 \\ \operatorname{sgn}\left(\mu^{*}(i)-\mu^{*}(j)\right) & \delta(i)=1, \delta(j)=1\end{cases}
$$


The scores for ranking 2 are defined similarly.

In the next section, we shall study a null hypotheses $H_{0}$ for testing independence between two random variables. We assume that the number of ranked observations in $\boldsymbol{\mu}^{*}$ and $\nu^{*}$ are fixed as are the tie patterns and the pattern of missing observations. Moreover, under $H_{0}$, the elements in $C_{\delta_{1}}\left(\boldsymbol{\mu}^{*}\right)$ are equally likely and are independent of those in $C_{\delta_{2}}\left(\boldsymbol{\nu}^{*}\right)$. Hence, it is easily shown that under $H_{0}$, the measures $A^{*}\left(\boldsymbol{\mu}^{*}, \boldsymbol{\nu}^{*}\right)$ for both Spearman and Kendall are conditional expectations given the classes of complete compatible rankings:

$$
A^{*}\left(\boldsymbol{\mu}^{*}, \boldsymbol{\nu}^{*}\right)=E\left[A(\boldsymbol{\mu}, \boldsymbol{\nu}) \mid C_{\delta_{1}}\left(\boldsymbol{\mu}^{*}\right), C_{\delta_{2}}\left(\boldsymbol{\nu}^{*}\right)\right] .
$$

As noted in Alvo and Cabilio (1995), this remark along with the fact that

$$
E\left(A_{K}-\frac{4}{t} A_{S}\right)^{2}=O\left(t^{2}\right)
$$

implies that the generalized test statistics are asymptotically equivalent as $t \rightarrow \infty$. Consequently, in what follows we shall be concerned only with the Spearman case.

\subsection{Asymptotic Distribution of $A_{S}^{*}$}

The generalized Spearman distance remains unchanged under any permutation relabeling of the items. This is a property known as right invariance (see Alvo and Cabilio, 1993). Assuming $k_{1} \leq k_{2}$, we may consequently relabel the items in such a way that in ranking 2 , the first $k_{2}$ objects are the one ranked and similarly tied items can be arranged arbitrarily among themselves in any sequence accordingly. Hence, the missing items can be placed at the end and the new rankings $\nu^{*}(j)$ appear in natural order in ranking 2. We let $o_{j}$ be the label of the $j^{\text {th }}$ item ranked in ranking 1 , and $k^{*}$ be the number of items ranked in ranking 1 among the $k_{2}$ ranked in ranking 2 . Define

$$
o_{j}^{*}= \begin{cases}\nu^{*}\left(o_{j}\right) & \text { if } 1 \leq j \leq k^{*} \\ \frac{k_{2}+1}{2} & \text { if } k^{*}+1 \leq j \leq k_{1},\end{cases}
$$

and $\bar{o}=\sum_{j=1}^{k_{1}} \frac{o_{j}^{*}}{k_{1}}$.

As an example, consider the following measurements $\left(X_{1}, X_{2}\right)$ from $t=10$ individuals.

\begin{tabular}{|l|cccccccccc|}
\hline$j$-th Individual & 1 & 2 & 3 & 4 & 5 & 6 & 7 & 8 & 9 & 10 \\
\hline$X_{2}$ & 12 & 17 & 17 & 17 & 24 & 29 & 33 & 35 & - & - \\
$X_{1}$ & 15 & 37 & - & 31 & 18 & 42 & - & 39 & - & 37 \\
\hline ranking $2 \nu^{*}(j)$ & 1 & 3 & 3 & 3 & 5 & 6 & 7 & 8 & - & - \\
ranking $1 \mu^{*}(j)$ & 1 & 4.5 & - & 3 & 2 & 7 & - & 6 & - & 4.5 \\
\hline
\end{tabular}

Here $k_{1}=7, k_{2}=8, k^{*}=6, o_{1}=1, o_{2}=2, o_{3}=4, o_{4}=5, o_{5}=6, o_{6}=8, o_{7}=10$, $o_{1}^{*}=1, o_{2}^{*}=3, o_{3}^{*}=3, o_{4}^{*}=5, o_{5}^{*}=6, o_{6}^{*}=8, o_{7}^{*}=4.5$.

For the analyses which follow, we shall focus on the similarity measure $A_{S}^{*}$. Following Lehmann $\left(1975\right.$, p. 360), let $U_{1}, \cdots, U_{k_{1}}$ be independent random variables uniformly 
distributed on $(0,1)$, let $R_{j}$ be the rank of $U_{j}\left(j=1, \cdots, k_{1}\right)$ and define the function $a_{k_{1}}(u)$ by

$$
k_{1} a_{k_{1}}(u)= \begin{cases}\frac{1}{2}\left(g_{11}+1\right) & \text { if } 0<u \leq \frac{g_{11}}{k_{1}} \\ g_{11}+\frac{1}{2}\left(g_{12}+1\right) & \text { if } \frac{g_{11}}{k_{1}}<u \leq \frac{g_{11}+g_{12}}{k_{1}} \\ \vdots & \vdots \\ \left(k_{1}-g_{1, e_{1}}\right)+\frac{1}{2}\left(g_{1, e_{1}}+1\right) & \text { if } \frac{k_{1}-g_{1, e_{1}}}{k_{1}}<u \leq 1\end{cases}
$$

Then the $k_{1}$ tuple $\left(a_{k_{1}}\left(\frac{R_{1}}{k_{1}}\right), \cdots, a_{k_{1}}\left(\frac{R_{k_{1}}}{k_{1}}\right)\right)$ and $\left(\frac{\mu^{*}\left(o_{1}\right)}{k_{1}}, \cdots, \frac{\mu^{*}\left(o_{k_{1}}\right)}{k_{1}}\right)$ have the same distribution. Re-write $A_{S}^{*}$ as

$$
\begin{aligned}
A_{S}^{*} & =\frac{(t+1)^{2}}{\left(k_{1}+1\right)\left(k_{2}+1\right)} \sum_{j=1}^{k_{1}}\left[o_{j}^{*}-\frac{k_{2}+1}{2}\right]\left[\mu^{*}\left(o_{j}\right)-\frac{k_{1}+1}{2}\right] \\
& =\frac{(t+1)^{2}}{\left(k_{1}+1\right)\left(k_{2}+1\right)} \sum_{j=1}^{k_{1}}\left[o_{j}^{*}-\bar{o}\right]\left[\mu^{*}\left(o_{j}\right)-\frac{k_{1}+1}{2}\right] \\
& =\frac{(t+1)^{2} k_{1}}{\left(k_{1}+1\right)\left(k_{2}+1\right)} \sum_{j=1}^{k_{1}}\left[o_{j}^{*}-\bar{o}\right] \frac{\mu^{*}\left(o_{j}\right)}{k_{1}} .
\end{aligned}
$$

It follows that under $H_{0}, A_{S}^{*}$ has the same distribution as $S_{k_{1}}$ where $S_{k_{1}}$ is a linear rank statistic of the form $S_{k_{1}}=\sum_{j=1}^{k_{1}} b_{j} a_{k_{1}}\left(\frac{R_{j}}{k_{1}}\right)$, with $b_{j}=\frac{(t+1)^{2} k_{1}}{\left(k_{1}+1\right)\left(k_{2}+1\right)}\left[o_{j}^{*}-\bar{o}\right]$.

Theorem 1 Assume that

(i) $k^{*} \rightarrow \infty$ (hence $k_{1} \rightarrow \infty, k_{2} \rightarrow \infty$ and $t \rightarrow \infty$ ) with $k^{*} / t \rightarrow \lambda>0$ (and hence $\lambda \leq \frac{k_{1}}{t}<1$ and $\left.\lambda \leq \frac{k_{2}}{t}<1\right)$

(ii) $\max _{j=1, \cdots, e_{1}} \frac{g_{1 j}}{k^{*}}$ is bounded away from 1;

(iii) $\max _{j=1, \cdots, e_{2}} \frac{g_{2 j}}{k^{*}}$ is bounded away from 1 .

Then under $H_{0}$, the distribution of $S_{k_{1}}$ is asymptotically normal as $k^{*} \rightarrow \infty$.

Proof: See the Appendix.

Using Theorem $1, A_{S}^{*}$ is also asymptotically normally distributed under $H_{0}$. Moreover, by applying Theorem 'a' on p. 160 of Hájek and Šidák (1967), it can be shown that under $H_{0}$, the expected value of $A_{S}^{*}$ is zero and the exact variance of $A_{S}^{*}$ is

$$
\begin{aligned}
\operatorname{Var}\left(A_{S}^{*}\right) & =\left[\frac{(t+1)^{2}}{\left(k_{1}+1\right)\left(k_{2}+1\right)}\right]^{2}\left(\frac{1}{k_{1}-1}\right) \sum_{j=1}^{k_{1}}\left(o_{j}^{*}-\bar{o}\right)^{2} \sum_{j=1}^{k_{1}}\left(\mu^{*}\left(o_{j}\right)-\frac{k_{1}+1}{2}\right)^{2} \\
& =\left[\frac{(t+1)^{2} k_{1}}{\left(k_{1}+1\right)\left(k_{2}+1\right)}\right]^{2} \frac{\sum_{j=1}^{k_{1}}\left(o_{j}^{*}-\bar{o}\right)^{2}}{12}\left\{1-\frac{\sum_{j=1}^{e_{1}}\left[g_{1 j}^{3}-g_{1 j}\right]}{k_{1}^{3}-k_{1}}\right\} .
\end{aligned}
$$




\subsection{Efficiency of the Test Statistic}

An important consideration in rank tests is the efficiency of the test statistic. In particular, we would like to compare the proposed statistic $A_{S}^{*}$ with the Spearman statistic obtained by discarding all the missing observations. It is shown below that under the location shift alternative to $H_{0}, A_{S}^{*}$ is always more powerful than the corresponding Spearman statistic based on the reduced sample.

Following the approach of Hájek and Sidák (1967), let $X_{1}, \cdots, X_{t}$ be independent random variables whose joint density under the location shift alternative to $H_{0}$ is given by $q_{\beta}=\prod_{j=1}^{t} f_{0}\left(x_{j}-\beta_{j}\right)$ where $f_{0}$ is a known density function having finite Fisher information $I\left(f_{0}\right)$ and $\boldsymbol{\beta}=\left(\beta_{1}, \cdots, \beta_{t}\right)$ is an arbitrary vector. Upon deletion of all pairs with missing values, we let $k_{2}=t$, and $k_{1}=k$ where $k$ is simply the actual number of $X$ 's observed. Therefore, the Spearman type statistic based on the reduced sample can be written in the form

$$
\bar{A}_{S}=\frac{(t+1)^{2}}{k+1} \sum_{j=1}^{k}\left[o_{j}^{\#}-\frac{k+1}{2}\right]\left[\frac{\mu^{*}\left(o_{j}\right)}{t+1}\right]
$$

where $o_{j}^{\#}$ is defined as the midrank of the $j^{\text {th }}$ item ranked in ranking 1 . The statistic (3) can be expressed as

$$
A_{S}^{*}=\frac{(t+1)^{2}}{k+1} \sum_{j=1}^{k}\left[\nu^{*}\left(o_{j}\right)-\bar{o}\right]\left[\frac{\mu^{*}\left(o_{j}\right)}{t+1}\right]
$$

since $k=k_{1}=k^{*}$ and $o_{j}^{*}=\nu^{*}\left(o_{j}\right)$. On setting $\bar{\beta}=\sum_{i=1}^{t} \beta_{i} / t$ and provided that

$$
\max _{1 \leq i \leq t}\left(\beta_{i}-\bar{\beta}\right)^{2} \rightarrow 0 \quad \text { and } \quad I\left(f_{0}\right) \sum_{i=1}^{t}\left(\beta_{i}-\bar{\beta}\right)^{2} \rightarrow b^{2} \text { for } 0<b^{2}<\infty
$$

it follows immediately that, under the alternative $q_{\beta}$, both $\bar{A}_{S}$ and $A_{S}^{*}$ are asymptotically normal with means and variances given respectively by

$$
\begin{aligned}
E\left[\bar{A}_{S} \mid q_{\beta}\right] & =\frac{(t+1)^{2}}{k+1} \sum_{j=1}^{k}\left(o_{j}^{\#}-\frac{k+1}{2}\right)\left(\beta_{o_{j}}-\bar{\beta}\right) \int_{0}^{1} u \phi\left(u, f_{0}\right) d u, \\
E\left[A_{S}^{*} \mid q_{\beta}\right] & =\frac{(t+1)^{2}}{k+1} \sum_{j=1}^{k}\left(\nu^{*}\left(o_{j}\right)-\bar{o}\right)\left(\beta_{o_{j}}-\bar{\beta}\right) \int_{0}^{1} u \phi\left(u, f_{0}\right) d u \\
\operatorname{Var}\left[\bar{A}_{S} \mid q_{\beta}\right] & =\frac{(t+1)^{4}}{12(k+1)^{2}} \sum_{j=1}^{k}\left[o_{j}^{\#}-\frac{k+1}{2}\right]^{2} \\
\operatorname{Var}\left[A_{S}^{*} \mid q_{\beta}\right] & =\frac{(t+1)^{4}}{12(k+1)^{2}} \sum_{j=1}^{k}\left[\nu^{*}\left(o_{j}\right)-\bar{o}\right]^{2} .
\end{aligned}
$$

Here $\phi\left(u, f_{0}\right)=\left[f^{\prime}\left(F^{-1}(u)\right)\right] /\left[f\left(F^{-1}(u)\right)\right]$ for $0<u<1$, and $F$ is the cumulative distribution function of $f$. 
Moreover, the asymptotic efficiencies for $\bar{A}_{S}$ and $A_{S}^{*}$ can be obtained as

$$
\begin{aligned}
& e_{\bar{A}_{S}}=\lim \frac{\left[\sum_{j=1}^{k}\left(o_{j}^{\#}-\frac{k+1}{2}\right)\left(\beta_{o_{j}}-\bar{\beta}\right)\right]^{2}}{\sum_{j=1}^{k}\left(o_{j}^{\#}-\frac{k+1}{2}\right)^{2} \sum_{j=1}^{t}\left(\beta_{j}-\bar{\beta}\right)^{2}} Q_{1}, \\
& e_{A_{S}^{*}}=\lim \frac{\left[\sum_{j=1}^{k}\left(\nu^{*}\left(o_{j}\right)-\bar{o}\right)\left(\beta_{o_{j}}-\bar{\beta}\right)\right]^{2}}{\sum_{j=1}^{k}\left(\nu^{*}\left(o_{j}\right)-\bar{o}\right)^{2} \sum_{j=1}^{t}\left(\beta_{j}-\bar{\beta}\right)^{2}} Q_{1}
\end{aligned}
$$

where $Q_{1}$ is a positive function of $f_{0}$ and the limit is taken as $t \rightarrow \infty, k \rightarrow \infty$ with $k / t \rightarrow$ $\lambda>0$. Therefore, the asymptotic relative efficiency of $A_{S}^{*}$ relative to $\bar{A}_{S}$ is given by $e_{A_{S}^{*}} / e_{\bar{A}_{S}}$. Consider the case where $\beta_{o_{j}}=\nu^{*}\left(o_{j}\right)(j=1, \cdots, k)$ and the remaining $\beta_{j}$ 's are arbitrary. This situation includes alternatives of the form $E\left(X_{i}\right)=\psi_{0}+\psi_{1} R\left(X_{i}\right)$ for $\psi_{1}>$ 0 where $R\left(X_{i}\right)$ is just the midrank of item $j$. It can be seen that irrespective of the density $f_{0}$, the asymptotic relative efficiency of $A_{S}^{*}$ relative to $\bar{A}_{S}$ is given by $A R E\left(A_{S}^{*}, \bar{A}_{S}\right)=$ $\lim _{k \rightarrow \infty} R\left(k, \boldsymbol{\nu}^{*}\right)$ where

$$
R\left(k, \boldsymbol{\nu}^{*}\right)=\frac{\sum_{j=1}^{k}\left(o_{j}^{\#}-\frac{k+1}{2}\right)^{2} \sum_{j=1}^{k}\left(\nu^{*}\left(o_{j}\right)-\bar{o}\right)^{2}}{\left[\sum_{j=1}^{k}\left(o_{j}^{\#}-\frac{k+1}{2}\right)\left(\nu^{*}\left(o_{j}\right)-\bar{o}\right)\right]^{2}} \geq 1 .
$$

Note that $R\left(k, \nu^{*}\right)>1$ in most cases. One exception would be the case of no tied and no missing observations in which case both $A_{S}^{*}$ and $\bar{A}_{S}$ reduce to $A_{S}$.

As in Alvo and Cabilio (1995) we may illustrate the results of the calculation of this efficiency. Suppose that $t=19, k=7, o_{1}^{*}=1.5, o_{2}^{*}=8.5, o_{3}^{*}=8.5, o_{4}^{*}=10, o_{5}^{*}=11$, $o_{6}^{*}=18, o_{7}^{*}=19$; as well, $o_{1}^{\#}=1, o_{2}^{\#}=o_{3}^{\#}=2.5, o_{4}^{\#}=4, o_{5}^{\#}=5, o_{6}^{\#}=6, o_{7}^{\#}=7$. Then the ratio of the efficiencies is 1.088. On the other hand, if $o_{1}^{*}=1.5, o_{2}^{*}=8, o_{3}^{*}=9$, $o_{4}^{*}=10, o_{5}^{*}=11, o_{6}^{*}=12,, o_{7}^{*}=18.5$ and, $o_{1}^{\#}=1, o_{2}^{\#}=2, o_{3}^{\#}=3, o_{4}^{\#}=4, o_{5}^{\#}=$ $5, o_{6}^{\#}=6, o_{7}^{\#}=7$, then the ratio of the efficiencies is 1.163 .

\section{Opinion Survey Data-Revisited}

In this section, we apply the proposed Spearman rank test to the opinion survey data to test whether the level of satisfaction of the Policy Address of the Chief Executive depends on the age of the respondents. For benchmark comparison, we consider a reduced sample whereby we discard all the observations with at least one missing variable and apply the classical Spearman rank test. The values of the test statistics and their $p$-values are tabulated in Table 2.

From the results shown in Table 2, it is seen that the $p$-values of the two tests do not give consensus results. At the 5\% significance level, the test based on the reduced sample rejects $H_{0}$ but the test based on the complete sample does not reject $H_{0}$. This indicates that the proposed test provides a simple way of handling the missing values in a fair manner such that the information carried in the partially missing observations is also utilized. As the standardized statistic is negative, this implies that $A_{S}^{*}$ is below its expected value and 
Table 2: Results of the analyses

\begin{tabular}{|c|c|c|c|}
\hline Test based on & $A_{S}^{*}$ & Standardized statistic & $p$-value \\
\hline reduced sample & -107165.00 & -2.2039 & 0.0276 \\
\hline complete sample & -210504.34 & -1.8812 & 0.0600 \\
\hline
\end{tabular}

hence indicates a negative association between age and the level of satisfaction. That is, young people tend to be less satisfied with the Policy address. We also performed a test of independence using a contingency table analysis of the same data whereby the "missing" categories for age and response were dropped. The row corresponding to response " 1 " was also dropped since there are no occurrences. The chi-square statistic based on 12 degrees of freedom yielded a value of 15.806 and the corresponding p-value is 0.200.

\section{Concluding Remarks}

Rank tests are widely applicable in many contexts. However, one main disadvantage of the rank tests is that they may not be applicable when the data contain missing observations and/or tied values. The problem appears very often in two-way ordinal classifications used in analyzing survey data. In this paper, we proposed a rank test for independence which is a generalization of the Spearman rank correlation based on a natural extension of the concept of distance between two incomplete rankings in order to include data consisting of both missing observations as well as ties. The test is simple and easily applicable. The test statistic reduces to the classical Spearman/Kendall rank statistic when there are no missing values; it reduces to the test proposed by Alvo and Cabilio (1995) when there are missing values but no ties.

Sometimes, we might want to have a measure of association to indicate the direction of influence between the two characteristics if the test for independence is rejected. It is easy to do so by defining a correlation measure in terms of the generalized Spearman distance $d_{S}^{*}\left(\boldsymbol{\mu}^{*}, \boldsymbol{\nu}^{*}\right)=c_{S}-A_{S}^{*}\left(\boldsymbol{\mu}^{*}, \boldsymbol{\nu}^{*}\right)$ between $\boldsymbol{\mu}^{*}$ and $\boldsymbol{\nu}^{*}$ as

$$
\alpha^{*}\left(\boldsymbol{\mu}^{*}, \boldsymbol{\nu}^{*}\right)=1-\frac{2\left(d^{*}-m\right)}{M-m}
$$

where $M$ and $m$ be the maximum and minimum value of the generalized Spearman distance $d^{*}$ taken over all possible patterns of the missing and tied observations when the number of tied groups in rankings 1 and 2, $e_{1}$ and $e_{2}$, respectively are fixed. Note that $-1 \leq \alpha^{*} \leq 1$. The calculations of $M$ and $m$ are not straightforward and this interesting problem is worthwhile for future research. 


\section{APPENDIX: Proof of Theorem 1}

From Lehmann (1975, Corollary 4 on p. 358), if

$$
\frac{\max _{i}\left(o_{i}^{*}-\bar{o}\right)^{2}}{\sum_{i=1}^{k_{1}}\left[o_{i}^{*}-\bar{o}\right]^{2} / k_{1}} \quad \text { is bounded as } k_{1} \rightarrow \infty
$$

and the functions $a_{k_{1}}$ satisfy the following three conditions:

(A.1) there exist constants $-\infty<m<M<\infty$ such that $m \leq a_{k_{1}}(u) \leq M$ for all $u$ and all $k_{1}$;

(A.2) the variance $\operatorname{Var}\left[a_{k_{1}}(U)\right]$ are bounded away from zero;

(A.3) the expectations $E\left[a_{k_{1}}\left(U_{1}\right)-a_{k_{1}}\left(\frac{R_{1}}{k_{1}}\right)\right]^{2}$ tend to zero;

then $S_{k_{1}}$ is asymptotically normally distributed.

Let $\bar{o}^{*}=\sum_{j=1}^{k^{*}} \frac{o_{j}^{*}}{k^{*}}$. Note that

$$
\begin{aligned}
\sum_{i=1}^{k_{1}}\left[o_{i}^{*}-\bar{o}\right]^{2} & =\sum_{i=1}^{k^{*}}\left[\nu^{*}\left(o_{i}\right)-\bar{o}^{*}\right]^{2}+k^{*}\left(\bar{o}^{*}-\bar{o}\right)^{2}+\left(k_{1}-k^{*}\right)\left[\frac{k_{2}+1}{2}-\bar{o}\right]^{2} \\
& \geq \sum_{i=1}^{k^{*}}\left[\nu^{*}\left(o_{i}\right)-\bar{o}^{*}\right]^{2} \geq \frac{1}{12}\left[k^{*}\left(k^{* 2}-1\right)-\sum_{i=1}^{e_{2}} g_{2 i}^{*}\left(g_{2 i}^{*}-1\right)\right] \\
& =\frac{1}{12}\left(k^{* 3}-\sum_{i=1}^{e_{2}} g_{2 i}^{* 3}\right) .
\end{aligned}
$$

Moreover, $\left(o_{i}^{*}-\bar{o}\right)^{2} \leq(t-1)^{2}$. Hence,

$$
\begin{aligned}
\frac{\max _{i}\left(o_{i}^{*}-\bar{o}\right)^{2}}{\sum_{i=1}^{k_{1}}\left[o_{i}^{*}-\bar{o}\right]^{2} / k_{1}} & \leq \frac{12(t-1)^{2} k_{1}}{k^{*^{3}}-\sum_{i=1}^{e_{2}} g_{2 i}^{* 3}} \\
& <\frac{12}{\left(\frac{k^{*}}{t}\right)^{3}-\sum_{i=1}^{e_{2}}\left(\frac{g_{2 i}^{*}}{t}\right)^{3}}=\frac{12}{\left(\frac{k^{*}}{t}\right)^{3}\left[1-\sum_{i=1}^{e_{2}}\left(\frac{g_{2 i}^{*}}{k^{*}}\right)^{3}\right] .}
\end{aligned}
$$

Since $\max \left(g_{2 i} / k^{*}\right)$ is bounded away from 1 as $k^{*} \rightarrow \infty$, there exists an $\epsilon_{2}\left(0<\epsilon_{2}<1\right)$ such that $g_{2 i} \leq\left(1-\epsilon_{2}\right) k^{*}$ for all $i$. Therefore,

$$
\frac{\max _{i}\left(o_{i}^{*}-\bar{o}\right)^{2}}{\sum_{i=1}^{k_{1}}\left[o_{i}^{*}-\bar{o}\right]^{2} / k_{1}}<\frac{12}{\lambda^{3}\left[1-\left(1-\epsilon_{2}\right)^{2} \sum_{i=1}^{e_{2}} \frac{g_{2 i}^{*}}{k^{*}}\right]}=\frac{12}{\lambda^{3}\left[1-\left(1-\epsilon_{2}\right)^{2}\right]}
$$

and hence, the above condition is satisfied. Further, condition (A.1) is obviously satisfied with $m=0$ and $M=1$. As to condition (A.2), note that from (2), $k_{1} a_{k_{1}}$ are just the 
midranks of the observations in ranking 1, and it is well known (see Lehmann 1975, p. 294) that

$$
\operatorname{Var}\left[a_{k_{1}}(U)\right]=\left(\frac{1}{12 k_{1}^{2}}\right)\left[\left(k_{1}^{2}-1\right)-\frac{\sum_{i=1}^{e_{1}} g_{1 i}\left(g_{1 i}^{2}-1\right)}{k_{1}}\right]=\frac{1}{12}\left[1-\sum_{i=1}^{e_{1}}\left(\frac{g_{1 i}}{k_{1}}\right)^{3}\right]
$$

By an argument similar to the above with $0<\epsilon_{1}<1$, such that $g_{1 i} \leq\left(1-\epsilon_{1}\right) k_{1}$ for all $i$, we have

$$
\operatorname{Var}\left[a_{k_{1}}(U)\right] \geq \frac{1}{12}\left[1-\left(1-\epsilon_{1}\right)^{2}\right]>0
$$

which shows that condition (A.2) is also satisfied.

To show that condition (A.3) is satisfied, recall the definition of $R_{1}$ and let $I_{h}=1$ if $0<u<\sum_{i=1}^{h} g_{1 i} / k_{1}$ or $I_{h}=0$ otherwise for $h=1, \cdots, e_{1} ; J_{h}=1$ if $0<\frac{R_{1}}{k_{1}} \leq$ $\sum_{i=1}^{h} g_{1 i} / k_{1}$ or $J_{h}=0$ otherwise for $h=1, \cdots, e_{1}$ and $a_{k_{1}}^{(h)}(u)=1$ if $\sum_{i=1}^{h-1} g_{1 i} / k_{1}<u \leq$ $\sum_{i=1}^{h} g_{1 i} / k_{1}$ for $h=1, \cdots, e_{1}$. It can be seen that

$$
a_{k_{1}}(u)=\frac{1}{2 k_{1}}\left(g_{11}+1\right) a_{k_{1}}^{(1)}(u)+\cdots+\frac{1}{k_{1}}\left[\left(k_{1}-g_{1, e_{1}}\right)+\frac{1}{2}\left(g_{1, e_{1}}+1\right)\right] a_{k_{1}}^{\left(e_{1}\right)}(u) .
$$

From the inequality $\left(X_{1}+\cdots+X_{e_{1}}\right)^{2} \leq e_{1}\left(X_{1}^{2}+\cdots+X_{e_{1}}^{2}\right)$ and since the coefficients of $a_{k_{1}}^{(j)}(u)$ are all less than or equal to 1 , it follows that

$$
0 \leq\left[a_{k_{1}}\left(U_{1}\right)-a_{k_{1}}\left(\frac{R_{1}}{k_{1}}\right)\right]^{2} \leq e_{1} \sum_{i=1}^{e_{1}}\left[a_{k_{1}}^{(i)}\left(U_{1}\right)-a_{k_{1}}^{(i)}\left(\frac{R_{1}}{k_{1}}\right)\right]^{2}
$$

Note that $\sum_{j}^{k_{1}}\left[a_{k_{1}}^{(1)}\left(U_{j}\right)-a_{k_{1}}^{(1)}\left(\frac{R_{j}}{k_{1}}\right)\right]^{2}=\left|W-g_{11}\right|$ where $W$ is the number of $U$ 's less than or equal to $g_{11} / k_{1}$, the variables $\left[a_{k_{1}}^{(1)}\left(U_{j}\right)-a_{k_{1}}^{(1)}\left(\frac{R_{j}}{k_{1}}\right)\right]$ are independently and identically distributed with mean 0 , and $W$ has the binomial distribution with $k_{1}$ trials and probability of success $g_{11} / k_{1}$. Consequently, we have

$$
E\left[a_{k_{1}}^{(1)}\left(U_{1}\right)-a_{k_{1}}^{(1)}\left(\frac{R_{1}}{k_{1}}\right)\right]^{2}=\frac{E\left|W-d_{11}\right|}{k_{1}} \leq \sqrt{\frac{1}{k_{1}} \frac{g_{11}}{k_{1}}\left(1-\frac{g_{11}}{k_{1}}\right)} \rightarrow 0
$$

as $k_{1} \rightarrow \infty$ and $g_{11} / k_{1}$ is bounded away from 1. Similarly, for $h<e_{1}$,

$$
\begin{aligned}
0 & \leq E\left[a_{k_{1}}^{(h+1)}\left(U_{1}\right)-a_{k_{1}}^{(h+1)}\left(\frac{R_{1}}{k_{1}}\right)\right]^{2} \\
& =E\left[I_{h+1}-I_{h}-J_{h+1}+J_{h}\right]^{2} \\
& \leq E\left[I_{h+1}-J_{h+1}\right]^{2}+E\left[I_{h}-J_{h}\right]^{2} \rightarrow 0 \quad \text { as } k_{1} \rightarrow \infty
\end{aligned}
$$

Hence, we have $E\left[a_{k_{1}}\left(U_{1}\right)-a_{k_{1}}\left(\frac{R_{1}}{k_{1}}\right)\right]^{2} \rightarrow 0$ as $k_{1} \rightarrow \infty$ and this completes the proof. 


\section{Acknowledgments}

The research of Philip L.H. Yu and K.F. Lam was supported by the CRCG grant 337/017/0014 of the University of Hong Kong and partially supported by a grant from the Research Grants Council of the Hong Kong Special Administrative Region, China (Project No. HKU 7169/98H), whereas the research of M. Alvo was supported by the Natural Sciences and Engineering Council of Canada Grant OGP0009068. The authors would like to thank the Social Sciences Research Centre of the University of Hong Kong for providing the data set.

\section{References}

M. Alvo and P. Cabilio. Rank correlations and the analysis of rank-based experimental design. In M.A. Flinger and S.J. Verducci, editors, Probability Models and Statistical Analyses for Ranked Data. Lecture Notes in Statistics, volume 80, pages 140-154. Springer, New York, 1993.

M. Alvo and P. Cabilio. Rank correlation methods for missing data. The Canadian J. of Statistics, 23(4):345-358, 1995.

J. Hájek and Z. Šidák. Theory of Rank Tests. Academic Press, New York, 1967.

M.G. Kendall. A new measure of rank correlation. Biometrika, 30:81-93, 1938.

E.L. Lehmann. Nonparametrics: Statistical Methods Based on Ranks. Holden-Day, San Francisco, 1975.

C. Spearman. The proof and measurement of association between two things. Am. J. of Psychol., 15:72-101, 1904.

Authors' addresses:

Philip L.H. Yu

K.F. Lam

Department of Statistics and Actuarial Sci-

ence

The University of Hong Kong

Pokfulam Road

Hong Kong

Tel.: (852) 2857-8321

Fax: (852) 2858-9041

E-mail: plhyu@hku.hk
Mayer Alvo

Department of Mathematics and Statistics

University of Ottawa

585 King Edward Ave.

P.O. Box 450, Stn. A

Ottawa, Ontario K1N 6N5

Canada

Tel.: (613) 562-5864

Fax: (613) 562-5776

E-mail: MALVO@science.uottawa.ca 